Research Article

\title{
Plasmon Mediation of Charge Pairing in High Temperature Superconductors
}

\author{
Abel Mukubwa $(D)$ and John Wanjala Makokha \\ Department of Science Technology and Engineering, Kibabii University, P.O Box 1699-50200, Bungoma, Kenya \\ Correspondence should be addressed to Abel Mukubwa; abelmuwa@gmail.com
}

Received 7 July 2021; Accepted 14 December 2021; Published 28 December 2021

Academic Editor: Sergio Ulloa

Copyright (C) 2021 Abel Mukubwa and John Wanjala Makokha. This is an open access article distributed under the Creative Commons Attribution License, which permits unrestricted use, distribution, and reproduction in any medium, provided the original work is properly cited.

\begin{abstract}
A Bose-Einstein condensate (BEC) of a nonzero momentum Cooper pair constitutes a composite boson or simply a boson. We demonstrated that the quantum coherence of the two-component BEC (boson and fermion condensates) is controlled by plasmons. It has been proposed that plasmons, observed in both electron-doped and hole-doped cuprates, originates from the long-range Coulomb screening, where the transfer momentum $|\mathbf{q}| \longrightarrow 0$. We further show that the screening mediates bosonfermion pairing at condensate state. While only about $1 \%$ of plasmon energy mediates the charge pairing, most of the plasmon energy is used to overcome the modes that compete against superconductivity such as phonons, charge density waves, antiferromagnetism, and damping effects. Additionally, the dependence of frequency of plasmons on the material of a superconductor is also explored. This study gives a quantum explanation of the modes that enhance and those that inhibit superconductivity. The study informs the nature of electromagnetic radiations (EMR) that can enhance the critical temperature of such materials.
\end{abstract}

\section{Introduction}

A system that contains Cooper-pair bosons interacting with electrons (fermions) in Bose-Einstein condensate (BEC) state was first proposed by Tolmachev [1]. Different from ultracold atom systems, which form condensates near absolute zero temperature, Cooper-pair bosons together with fermions undergo first-order transition from the nematic (BCS) state to BEC state at $T_{c}$, which is much higher than absolute zero $[2,3]$. Recent studies have explored fermion [4] and atom-mediated [5] pairing between BEC systems. However, Pasuparthy et al. [6] have suggested that the strength of pairing in high temperature superconductors is determined by the unusual electronic excitations. Some time back, it was predicted [7] that high temperature superconductivity (HTSC) arises from the coexistence of phonon and plasmon mechanisms. The fact is that Cooper pairs play a key role in HTSC already caters, to a large extend, for the phonon mechanism. Plasmon mediation in electronic systems such as Cooper pairs causes transition to a superconducting state with a relatively high $T_{c}[8,9]$. Excitation of charges with energy much below that of the Fermi electron-acoustic plasmons have been observed in both electron and hole-doped cuprates using resonant inelastic X-ray scattering (RIXS) $[10,11]$. The observed acoustic plasmons are predominantly associated with $\mathrm{O}$ sites of the copper oxide plane [12]. Plasmons in HT superconductors originate from singularity of long-range Coulomb interactions in the limit of long wavelength [13]. This scenario depends on a charged particle moving in the electron plasma under the influence of an external field/force. In the long wavelength plasmon branch where $q \longrightarrow 0$, Coulomb screening by the Thomas-Fermi method may be considered [14]. The large charge fluctuations (plasmons) in cuprates contribute significantly to the pairing mechanism [15] and lead to large and sharp velocity peak at $k=k_{F}$, which persists in the presence of screening [16].

The plasmon dispersions observed in YBCO are anisotropic and quadratic in ab-plane. Strongly anisotropic dielectric functions have also been observed [17]. While surface-plasmon dispersion at the interface of two media are isotropic, bulk-plasmon dispersions are anisotropic. The 
bulk-plasmon dispersion anisotropy increases with momentum transfer $q$ up to its critical value $q_{c}=\omega_{p} / v_{F}$ beyond which the anisotropy tends to zero. Here, $\omega_{p}=\left(3 / r_{s}^{3}\right)^{1 / 2}$ is the plasma frequency, $v_{F}=\left(3 \pi^{2} n_{0}\right)^{1 / 3}$ is the Fermi velocity, $n_{0}$ is the electron density, and $r_{s}=\left[3 /\left(4 \pi^{2} n_{0}\right)\right]^{1 / 3}$ is the bulk radius per electron $[18,19]$.

In high temperature superconductors, the number of Cooper pairs is negligible compared to that of free electrons and the bulk-plasmon dispersion is almost similar to that in pure metal-free electrons and follows the relation [19]

$$
E^{2}=\omega_{p}^{2}+\left[\frac{3}{5} v_{F}^{2}-\frac{v_{F}}{3 \pi}-\varepsilon\right] q^{2}+\frac{1}{4} q^{4}
$$

The term in $q^{2}$ has the kinetic energy contribution $\left(3 v_{F}^{2} / 5\right)$, the exchange interaction contribution $\left(v_{F} / 3 \pi\right)$, and the correlation energy contribution $(\varepsilon)$. Note that $\varepsilon<v_{F} / 3 \pi$. The term in $q^{4}$ is the quasifree-electron kinetic energy contribution. The screened of Coulomb potential is proportional to the critical temperature of HTSC [20].

Other than plasmons, charge density waves (CDW) also influence the formation bosons-fermion pair condensate at the ground state. Short-range CDW arises from strong correlations, but it remains unclear as to whether they strictly cooperate or compete with superconductivity; in this case, plasmonic waves mediate boson-fermion interaction [21, 22]. Charge density waves occur at significantly different wave vectors, and they develop in two stages [23]. First, a phonon-dependent CDW with quasicommensurate wave vector emerges at high temperature and forms along the $\mathrm{CuO}_{2}$ planes $[23,24]$. At low temperature, another CDW with strong doping dependent wave vector forms [23]. At optimal doping, CDW correlations show strong changes through the superconducting transition without discontinuity [25]. It has been argued that phonon-dependent CDW boost superconductivity based on the correlation with the largest electron-phonon coupling and lowest Fermi velocities at the same k-point [26]. On the contrary, doping-dependent CDW inhibits the formation of superconducting order by gapping the nested portion of the Fermi surface [27]. The competing CDW and superconducting phases have been observed in cuprates [28]. The precursor CDW and its phase mode are building blocks of highly intertwined electronic ground state in cuprates [23]. They determine the pairing strength between bosons and fermions in forming a pair condensate. The response of the low-lying $\mathrm{Ba}-5 \mathrm{p}$ and $\mathrm{Y}-4 \mathrm{p}$ core electrons is shown to interact strongly with $\mathrm{Cu}-3 \mathrm{~d}$ and $\mathrm{O}-2 \mathrm{p}$ excitations, with important consequences on screening. Thus, the base/ low-lying elements also contributes to the nature of plasmon dispersions in high temperature superconductors.

This study has focused on Coulomb screening of a Cooper-pair boson as it moves in an electron plasma under phonon mediation. Section 2.1 deals with the consequences of the Coulomb screening-BFP coherence length, while plasmon frequency is discussed in Section 2.2. Finally, the results are discussed in Section 3, and conclusions are made in Section 4.

\section{Theoretical Formulation}

2.1. Plasmon-Mediated Boson-Fermion Pairing. The dielectric function $\epsilon\left(\mathbf{r}, r^{\prime}, t-t^{\prime}\right)$ is in particular a key quantity for superconductivity and is probed often via its Fourier transform $\epsilon(\mathbf{q}, x 3 c 9$; $)$ in the limit of Thomas-Fermi approximations where the transferred momentum $|\mathbf{q}|=q \longrightarrow 0$ with long-range interactions $\left(\left|\mathbf{r}-r^{\prime}\right| \longrightarrow \infty\right)$ [29]. The inverse $\varepsilon^{-1}$ of the dielectric function measures the screening of the bare Coulomb repulsion and can directly indicate reverse screening (antiscreening) spatial regions where the interaction between two electrons is attractive rather than repulsive. In these regions, electrons pair up, giving rise to superconductivity [29]. Introducing $\mathbf{k}$ dependence is necessary to address the evident anisotropy in HTSC $[30,31]$, a relationship that has been proved theoretically [32]. Thus, the general new dielectric function becomes

$$
\epsilon(\mathbf{q}, x 3 c 9 ;, \mathbf{k})=\epsilon(\mathbf{q})+\epsilon(x 3 c 9 ;)+\epsilon(\mathbf{k}) .
$$

Here, the dielectric function $\epsilon(\mathbf{q})$ represents the plasmon dispersion, $\epsilon(\omega)$ describes the collective excitation of the Fermi sea, while $\epsilon(\mathbf{k})$ represents the Coulomb screening of a boson by fermions during interaction. Both $\epsilon(\mathbf{q})$ and $\varepsilon(x 3 c 9 ;)$ arise from the plasmon dispersion relation in equation (1). It has also been noted that dielectric screening at shorter distances and at frequencies of the order of the superconducting gap, but small compared to the Fermi energy, can significantly enhance the transition temperature $T_{c}$ of an unconventional superconductor [33].

A plasmon is quantum of plasma oscillations and may be excited by passing a charged particle through plasma [34]. When a Cooper-pair boson is excited (by phonons), it displaces the electrons in its way due to Coulomb repulsion, thereby exciting a plasmon in the electron plasma. Thus, the boson creates a plasmon, due to Coulomb interaction, while the electron annihilates it. The first electron in the Cooper pair creates a phonon, while the second one creates a strong plasmon as it annihilates the phonon. Thus, the effective induced charge by a single boson is $+q$ (a hole). The boson is an impurity within the electron plasma with an associated screened Coulomb potential, $\psi(\mathrm{r})$, over distance $r$.

The function $\epsilon(\mathbf{k})$ relates the following: (i) the density $\rho_{h}(k)$ of holes induced in the electron plasma and the charge density $\rho_{b}(k)$ of bosons due to the phonon field and (ii) the screened potential $\psi(k)$, the unscreened potential $\psi_{b}(k)$, due to phonons and the unscreened potential $\psi_{b}(k)$, due to induced holes according to the following equation:

$$
\epsilon(k)=\frac{\psi_{b}(k)}{\psi(k)}=\frac{\rho_{b}(k)}{\rho(k)}=1-\frac{\rho_{h}(k)}{\rho(k)},
$$

where $\psi(k)=\psi_{b}(k)+\psi_{h}(k)$ and $\rho(k)=\rho_{h}(k)+\rho_{b}(k)$ are the total electrostatic potential due to the displacement of the fermion gas and the total charge density, respectively. When $\psi(k)>0$, the function $\epsilon(k)$ has a scattering effect, but when $\psi(k)<0$, the interacting particles experience attraction towards each other. 
The boson is a charge due to the external field (phonons), and it induces a positive charge in electron plasma. The screened Coulomb potential, $\psi(r)$, at a distance $r$ from charge $Q$ of a particle, has been determined as [34]

$$
\psi_{0}(r)=\frac{Q}{4 \pi \epsilon_{0} r} \exp \left(-\left|k_{s} r\right|\right),
$$

where

$$
k_{s}= \pm\left(\frac{3 n_{s} Q}{2 \epsilon_{0} \epsilon_{F}}\right)^{1 / 2}= \pm\left(\frac{4}{a_{0}}\left(\frac{3 n_{0}}{\pi}\right)^{1 / 3}\right)^{1 / 2} .
$$

Here, $1 / k_{s}$ is the Thomas-Fermi screening length, $a_{0}=$ $5.29 \times 10^{-11} \mathrm{~m}$ is the Bohr radius, and $n_{s}$ is the electron density. Since the d-electrons in copper play a central role in charge pairing, we choose to use its electron density, $8.5 \times$ $10^{22} \mathrm{~cm}^{-3}$ [34], while that of iron (for iron-based superconductors) is $17 \times 10^{22} \mathrm{~cm}^{-3}$. Generally, among high temperature superconductors, cuprates are classified as group I superconductors, while iron pnictides as group II superconductors. In group I superconductors, the number density of particles per unit volume is dependent on temperature, while those in group II superconductors are independent of temperature of the material. In the case of a boson, $Q=-e$ (the second electron charge that creates the plasmon) and

$$
\psi_{0}(\varsigma)=-\frac{e}{4 \pi \epsilon_{0} r} \exp \left(-\left|k_{s} r\right|\right) .
$$

The total potential energy $V_{\text {plasmon }}$, which eventually leads to plasmon scattering, is equal to the screened Coulomb potential in equation (6) and so

$$
V_{\text {plasmon }}=\psi_{0}(\varsigma)=\frac{e^{2}}{4 \pi \epsilon_{0} r} \exp \left(-\left|k_{s} r\right|\right) .
$$

The potential $V_{\text {plasmon }}$ is attained at the nearest point where $r=r_{\min }$. Minimizing equation (7) with respect to $r$ yields

$$
\begin{aligned}
\frac{\mathrm{d} V_{\text {plasmon }}}{\mathrm{d} r}= & \frac{1}{r^{2}}\left(\frac{e^{2}}{4 \pi \epsilon_{0}}\right) \exp \left(-\left|k_{s} r\right|\right) \\
& +\frac{k_{s}}{r}\left(\frac{e^{2}}{4 \pi \epsilon_{0}}\right) \exp \left(-\left|k_{s} r\right|\right)=0 .
\end{aligned}
$$

Simplifying equation (8) leads to

$$
r= \begin{cases}\frac{1}{k_{s}}, & \text { minimum long }- \text { range limit, } \\ \infty, & \text { extreme long }- \text { range limit. }\end{cases}
$$

In the extreme long-range limit, the Coulomb screening cannot bring about boson-fermion pairing. However, at $r_{\text {min }}=\left|1 / k_{s}\right|$, the Coulomb screening leads to boson-fermion coherence. Since $r_{\min }>0$, then substituting for $k_{s}$ in equations (9) using (5) gives the minimum possible coherence length as

$$
\xi=\left[\frac{a_{0}}{4}\left(\frac{\pi}{3 n_{0}}\right)^{1 / 3}\right]^{1 / 2} .
$$

When $n_{0}$ is constant, $\xi$ is independent of the material. This is the maximum plasmon scattering energy attained (at the nearest point where $r=\xi$ ). The total potential energy, $V_{\text {plasmon }}$, due to the screening of the boson at $r=\xi$ is

$V_{\text {plasmon }}=\frac{e^{2}}{4 \pi \epsilon_{0} r} \exp (-1)=\frac{e^{2}}{2 \epsilon_{0}}\left[\frac{1}{a_{0}}\left(\frac{3 n_{0}}{\pi^{7}}\right)^{1 / 3}\right]^{1 / 2} \exp (-1)$.

For a constant $n_{0}, V_{\text {plasmon }}$ is the same for all materials. Considering the anisotropy in bulk-plasmon excitations, we express $r$ as a function and the average distance $r_{a v}$ as $r=r_{a v} \pm \delta r$. For a small anisotropy [29], $\lim _{\delta r \rightarrow 0}\left(\exp \left(-k_{s}\left[r_{a v} \pm \delta r\right]\right)\right) \approx \exp \left(-\left|k_{s} r\right|\right)=\exp (-1)$ and

$$
V_{\text {plasmon }}= \begin{cases}-\frac{e^{2}}{4 \pi \epsilon_{0} r_{\text {min }}^{u}} \exp (-1), & r>r_{\text {min }}, \\ -\frac{e^{2}}{4 \pi \epsilon_{0} r_{\text {min }}^{l}} \exp (-1), & r<r_{\text {min }},\end{cases}
$$

where, $r_{\min }^{l}<r_{\min }$ is the lower limit of $r_{\min }$ and $r_{\min }^{u}>r_{\min }$ is the upper limit of $r_{\min }$.

As the free electron (fermion) moves to annihilate the net plasmon, based on the Pauli exclusion principle, it experiences attraction towards the boson. The plasmon energy $V_{\text {plasmon }}$ is much greater than the attractive potential energy $V_{\mathrm{BFP}}$. Most of the plasmon energy is used to overcome the competing phonons, charge density waves (CDW), and the damping effect among others. Only a small fraction $\varrho$ of $V_{\text {plasmon }}$ remains to mediate boson-fermion interaction. The process of mediation mainly involves overcoming the Coulomb repulsion between the boson and the fermion. When $V_{\mathrm{BFP}}$ is greater than the Coulomb repulsion, the BFPC system $s$ is unbalanced, and therefore, the net effect is a reduced distance $r_{\min }$; the reduction in $r_{\min }$ does not necessarily translate to an increase in the plasmon potential energy, $V_{\text {plasmon }}$. When $V_{\text {BFP }}$ is compared to $\varrho V_{\text {plasmon }}$, the length $r_{\text {min }}$ becomes the size of the boson-fermion pair condensate. Now, using the averages,

$$
V_{\mathrm{BFP}}^{a v}=-\varrho V_{\text {plasmon }}^{a v}=-\frac{\varrho e^{2}}{4 \pi \epsilon_{0} r_{\min }} \exp (-1),
$$

or

$$
r_{\min }=-\frac{\varrho e^{2}}{4 \pi \epsilon_{0} V_{\mathrm{BFP}}^{a v}} \exp (-1) .
$$

The attractive potential energy $V_{\mathrm{BFP}}$ between a boson and a fermion has been given [35] as 


$$
V_{\mathrm{BFP}}= \begin{cases}-\frac{1}{2} E_{k}, & \text { maximum } V_{\mathrm{BFP}}, \\ -\frac{1}{3} E_{k}, & \text { minimum } V_{\mathrm{BFP}}\end{cases}
$$

where $E_{k}=2 k_{B} T_{c}$ is the excitation energy of a single-particle-like BFP condensate in a superconductor of critical temperature, $T_{c}$.

2.2. Frequency of a Plasmon. The energy $(E)$ contained in a plasmon of frequency $\omega$ can be obtained from the PlanckEinstein relation:

$$
E=-h \omega
$$

where $h=6.6262 \times 10^{-34}$ Js is Planck's constant. The negative sign has been used because the energy is attractive. The mediating plasmon is the source of attraction between the interacting boson and fermion in condensate state, and therefore,

$$
\omega=-\frac{V_{\mathrm{BFP}}}{h} .
$$

\section{Results and Discussion}

In Table 1 , the total plasmon energy ( $V_{\text {plasmon }}^{a v}$ ) (in equation (11)) has been compared to the mediation energy ( $V_{\mathrm{BFP}}^{a v}$ in equation (15)), and hence, the ratio $\varrho=V_{\mathrm{BFP}}^{a v} / V_{\text {plasmon }}^{a v}$ has also been determined. The mediation energy is equal to the attractive potential energy $\left(V_{\mathrm{BFP}}^{a v}\right)$, while the difference between $V_{\text {plasmon }}^{a v}$ and $V_{\text {BFP }}^{a v}$ has been referred to as the lost energy, $V_{\text {plasmon }}^{\text {lost }}$. The values in Table 1 are averages.

From Table 1, about 1\% or less of the plasmon energy mediates the boson-fermion interaction, while more than $99 \%$ is lost to competing modes such as phonons, charge density waves, and damping effects. The total plasmon energy obtained in this limit compares closely with the previous findings. For instance, Eremin and Kochergin [32] have noted that at small $\mathbf{q}$, the plasmon energy is about $0.3 \mathrm{eV}$ and grow with the wave vector up to $1 \mathrm{eV}$ at $\mathbf{q}=0.3 \pi$ and beyond [33]. Elsewhere, Bosovic [36] has noted that the plasmon energy at large $\mathbf{q}$ is $1-2 \mathrm{eV}$. Generally, the critical temperature $\left(\mathbf{T}_{\mathbf{c}}\right)$ is proportional to the plasmon mediation energy.

The coherence length of BFP condensate has been determined for various layered superconductors as given in Table 1 .

The coherence length of a BFP $\left(10^{-9} \mathrm{~m}\right)$ is much smaller than that of a BCS Cooper pair $\left(10^{-7} \mathrm{~m}\right)$. In HTSC, the coherence length is of the order of few interatomic distances [32], e.g., $1.5 \mathrm{~nm}$ in the ab-plane of $\mathrm{YBa}_{2} \mathrm{Cu}_{3} \mathrm{O}_{7-\delta}$ [36]. The results given in Table 2 are in agreement with these predictions. The coherence length for the BFP condensate is comparable to Thomas-Fermi length, which is in the order of $10^{-9} \mathrm{~m}$. The boson-fermion pair in iron pnictide superconductors has shorter coherence length than that in cuprate superconductors.

Note that the coherence length values in Table 2 denote the minimum possible distance for superconductive

\begin{tabular}{|c|c|c|c|c|}
\hline Superconductor & $T_{c}(K)$ & $V_{\mathrm{BFP}}^{a v}(\mathrm{meV})$ & $V_{\text {plasmon }}^{a v}(\mathrm{meV})$ & $\varrho(\%)$ \\
\hline \multicolumn{5}{|l|}{ Group I } \\
\hline $\mathrm{YBa}_{2} \mathrm{Cu}_{3} \mathrm{O}_{7}$ & 92 & 6.61 & 963.2 & 0.69 \\
\hline $\mathrm{Tl}_{2} \mathrm{Ba}_{2} \mathrm{CaCu}_{2} \mathrm{O}_{8}$ & 108 & 7.76 & 963.2 & 0.81 \\
\hline $\mathrm{Bi}_{2} \mathrm{Sr}_{2} \mathrm{CaCu}_{2} \mathrm{O}_{8}$ & 95 & 6.83 & 963.2 & 0.71 \\
\hline $\mathrm{HgBa}_{2} \mathrm{Ca}_{2} \mathrm{Cu}_{3} \mathrm{O}_{8}$ & 136 & 9.78 & 963.2 & 1.02 \\
\hline \multicolumn{5}{|l|}{ Group II } \\
\hline $\mathrm{HgBa}_{2} \mathrm{CuO}_{4}$ & 97 & 6.97 & 963.2 & 0.72 \\
\hline LiFeAs & 15 & 1.08 & 963.2 & 0.11 \\
\hline $\mathrm{BaFe}_{2}\left(\mathrm{As}_{0.7} \mathrm{P}_{0.3}\right)_{2}$ & 30 & 2.16 & 963.2 & 0.22 \\
\hline $\mathrm{Ca}_{0.33} \mathrm{Na}_{0.67} \mathrm{Fe}_{2} \mathrm{As}_{2}$ & 34 & 2.44 & 963.2 & 0.25 \\
\hline
\end{tabular}

TABle 1: Attractive potential energy (mediation energy) and plasmon energy.

TABLE 2: Coherence length $(\xi)$ of BFP condensate and plasmon frequency $(\omega)$ in layered superconductors.

\begin{tabular}{lcccc}
\hline Superconductor & $T_{c}(K)$ & $V_{\text {mediation }}^{a v}(\mathrm{meV})$ & $\xi(\mathrm{nm})$ & $\omega(\mathrm{THz})$ \\
\hline Group I & & & & \\
$\quad \mathrm{YBa}_{2} \mathrm{Cu}_{3} \mathrm{O}_{7}$ & 92 & 6.61 & 0.55 & 1.60 \\
$\mathrm{Tl}_{2} \mathrm{Ba}_{2} \mathrm{CaCu}_{2} \mathrm{O}_{8}$ & 108 & 7.76 & 0.55 & 1.87 \\
$\mathrm{Bi}_{2} \mathrm{Sr}_{2} \mathrm{CaCu}_{2} \mathrm{O}_{8}$ & 95 & 6.83 & 0.55 & 1.65 \\
$\mathrm{HgBa}_{2} \mathrm{Ca}_{2} \mathrm{Cu}_{3} \mathrm{O}_{8}$ & 136 & 9.78 & 0.55 & 2.36 \\
\hline $\mathrm{Group} \mathrm{II}$ & 97 & 6.97 & 0.55 & 1.68 \\
$\mathrm{HgBa}$ & $\mathrm{CuO}_{4}$ & 1.08 & 0.55 & 0.26 \\
$\mathrm{LiFeAs}_{\mathrm{BaFe}}\left(\mathrm{As}_{0.7} \mathrm{P}_{0.3}\right)_{2}$ & 30 & 2.16 & 0.55 & 0.52 \\
$\mathrm{Ca}_{0.33} \mathrm{Na}_{0.67} \mathrm{Fe}_{2} \mathrm{As}_{2}$ & 34 & 2.44 & 0.55 & 0.59 \\
\hline
\end{tabular}

coherence. It has been observed that the frequency of plasmons influences the absorption rate of infrared radiations $[37,38]$. The presence of terahertz plasmon frequencies maximizes the absorption of radiations of similar frequencies. Each material has a characteristic minimum frequency of a plasmon that can mediate the pairing between boson and fermion condensates in bulk superconductivity.

Other than the phenomenological BFPC model used in this work to study plasmon mediation of charge pairing, the full density functional theory (DFT) calculations could also be used to reveal the contribution of charge density waves to charge pairing in high temperature superconductors. Prior works on the use of full $a b$ initio calculations have been detailed in references [39, 40].

\section{Conclusion}

Plasmon energy in high temperature superconductors is sufficient to mediate for the high-energy pairing of charges at ground state. However, about $1 \%$ or less of the plasmon energy is used in the mediation, while the rest is used to counter the opposing modes such as CDW, plasmons, antiferromagnetism, and the damping effect. Consequently, the coherence length of a BFPC is found to be in the order of the Thomas-Fermi length, $10^{-9} \mathrm{~m}$.

\section{Data Availability}

There are no underlying datasets used to support the study. 


\section{Conflicts of Interest}

The authors declare that they have no conflicts of interest.

\section{References}

[1] V. V. Tomalchev, "Superconducting Bose-Einstein condensates of cooper pairs interacting with electrons," Physics Letters A, vol. 266, no. 4-6, pp. 400-408, 2000.

[2] Z. Cheng, "Bose-einstein condensation of nonideal cooper pairs in the Hartree-Fock-Popov theory," Foundations of Physics, vol. 46, no. 8, pp. 915-942, 2016.

[3] T. Hashimoto, Y. Ota, A. Tsuzuki et al., "Bose-Einstein condensation superconductivity induced by the disappearance of the nematic state," Science Advances, vol. 6, Article ID eabb9052, 2020.

[4] R. Liao, "Ultracold Bose mixtures with spin-dependent fermion-mediated interactions," Physical Review Research, vol. 2, Article ID 043218, 2020.

[5] Z. Li and L.-M. Kuang, "Controlling quantum coherence of a two-component Bose-Einstein condensate via an impurity atom," Quantum Information, vol. 19, p. 188, 2019.

[6] A. N. Pasupathy, A. Pushp, K. K. Gomes et al., "Electronic origin of the inhomogeneous pairing interaction in the high$T_{c}$ superconductor $\mathrm{Bi}_{2} \mathrm{Sr}_{2} \mathrm{CaCu}_{2} \mathrm{O}_{8+\delta}$," Science, vol. 320, no. 5873, pp. 196-201, 2008.

[7] V. Z. Kresin and Morawitz, "Plasmon and phonon mechanisms of superconductivity in layered high- $T_{c}$ cooper oxides," Physica C, vol. 153, pp. 1327-1328, 2002.

[8] E. A. Pashitskii and V. I. Pentegov, "On the plasmon mechanism of high- $T_{c}$ superconductivity in layered crystals and two-dimensional systems," Low Temperature Physics, vol. 34, no. 2, pp. 113-122, 2008.

[9] A. V. Matasov, "Characteristic lengths and plasmon superconductivity mechanism of some high temperature superconductors," in Proceedings of the 2019 Youth Conference on Radio Electronics, Electrical and Power Engineering, pp. 1-4, Moscow, Russia, March 2019.

[10] M. Hepting, L. Chaix, E. W. Huang et al., "Three-dimensional collective charge excitations in electron-doped copper oxide superconductors," Nature, vol. 563, no. 7731, pp. 374-378, 2018.

[11] A. Nag, M. Zhu, M. Bejas et al., "Detection of acoustic plasmons in hole-doped lanthanum and bismuth cuprate superconductors using resonant inelastic x-ray scattering," Physical Review Letters, vol. 125, Article ID 257002, 2018.

[12] K. Ishii, T. Tohyama, S. Asano et al., "Observation of momentum-dependent charge excitations in hole-doped cuprates using resonant inelastic $\mathrm{x}$-ray scattering at the oxygen $K$ edge," Physical Review B, vol. 96, no. 11, Article ID 115148, 2017.

[13] A. Greco, H. Yamase, and M. Bejas, "Origin of high-energy charge excitations observed by resonant inelastic X-ray scattering in cuprate superconductors," Communications on Physics, vol. 2, no. 1, p. 3, 2019.

[14] J. Bardeen, L. N. Cooper, and J. R. Schrieffer, "Theory of superconductivity," Physical Review, vol. 108, no. 5, pp. 1175-1204, 1957.

[15] D. Varshney, S. Shah, and R. K. Singh, "Specific heat studies in Ho-Ba-CuO superconductors: fermionic and bosonic contributions," Bulletin of Materials Science, vol. 23, no. 4, pp. 267-272, 2000.
[16] M. Weger and L. Burlachkov, "Reduced screening at the Fermi surface of high- $T_{c}$ cuprates," Physica C: Superconductivity, vol. 235-240, pp. 2387-2388, 1994.

[17] M. Knupfer, G. Roth, J. Fink, J. Karpinski, and E. Kaldis, "Plasmon dispersion and dielectric function in $\mathrm{YBa}_{2} \mathrm{Cu}_{4} \mathrm{O}_{8}$ single crystals," Physica C, vol. 230, pp. 1-2, 1994.

[18] J. Sprösser-Prou, A. Vom Felde, and J. Fink, "Aluminum bulkplasmon dispersion and its anisotropy," Physical Review B, vol. 40, no. 8, pp. 5799-5801, 1989.

[19] L. Serra, F. Garcias, M. Barranco, N. Barberán, and J. Navarro, "Bulk-plasmon dispersion relations in metals," Physical Review B, vol. 44, no. 4, pp. 1492-1498, 1991.

[20] S. Teranishi, K. Nishiguchi, and K. Kusakabe, "Material-dependent screening of Coulomb interaction in single-layer cuprates," Journal of the Physical Society of Japan, vol. 87, no. 11, Article ID 114701, 2018.

[21] J. Q. Lin, H. Miao, D. G. Mazzone et al., "Strongly correlated charge density wave in $\mathrm{La}_{2}-\mathrm{xSrxCuO}_{4}$ evidenced by dopingdependent phonon anomaly," Physical Review Letters, vol. 124, no. 20, Article ID 207005, 2020.

[22] A. Chikina, A. Fedorov, D. Bhoi et al., "Turning chargedensity waves into Cooper pairs," NPJ Quantum Materials, vol. 5, no. 1, p. 22, 2020.

[23] H. Miao, R. Fumagalli, M. Rossi et al., "Formation of incommensurate charge density waves in cuprates," Physical Review X, vol. 9, Article ID 031042, 2019.

[24] C. Di Catro, "Revival of charge density waves and charge density fluctuations in cuprate high temperature superconductors," Condensed Matter, vol. 5, no. 4, p. 70, 2020.

[25] H. Miao, G. Fabbris, R. J. Koch et al., "Charge density waves in cuprate superconductors beyond the critical doping," Nature Partner Journals, vol. 6, 2006.

[26] T. Kiss, T. Yokoya, A. Chainani et al., "Charge-order-maximized momentum-dependent superconductivity," Nature Physics, vol. 3, no. 10, pp. 720-725, 2007.

[27] S. V. Borisenko, A. A. Kordyuk, V. B. Zabolotnyy et al., "Two energy gaps and fermi-surface "arcs" in $\mathrm{NbSe}_{2}$," Physical Review Letters, vol. 102, no. 16, Article ID 166402, 2009.

[28] B. Leridon, S. Caprara, J. Vanacken et al., "Protected superconductivity at the boundaries of charge-density-wave domains," New Journal of Physics, vol. 22, no. 7, Article ID 073025, 2020.

[29] S. Huotari, J. Aleksi Soininen, G. Vankó, G. Monaco, and $\mathrm{V}$. Olevano, "Screening in $\mathrm{YBa}_{2} \mathrm{Cu}_{3} \mathrm{O}_{7-\delta}$ at large wave vectors," Physical Review B, vol. 82, Article ID 064514, 2010.

[30] M. Civelli, M. Capone, S. S. Kancharla, O. Parcollet, and G. Kotliar, "Dynamical breakup of the Fermi surface in a doped mott insulator," Physical Review Letters, vol. 95, no. 10, Article ID 106402, 2005.

[31] M. Civelli, "Doping-driven evolution of the superconducting state from a doped Mott insulator: cluster dynamical meanfield theory," Physical Review B, vol. 79, no. 19, Article ID 195113, 2009.

[32] M. V. Eremin and D. S. Kochergin, "On the theory of plasmon dispersion in electron-doped cuprates," JETP Letters, vol. 108, no. 2, pp. 132-136, 2018.

[33] S. Raghu, R. Thomale, and T. H. Geballe, "Optimal $T_{c}$ of cuprates: the role of screening and reservoir layers," Physical Review B, vol. 86, Article ID 094506, 2012.

[34] C. Kittel, Introduction to Solid State Physicsp. 117, 7th edition, Wiley, New York, NY, USA, 2004.

[35] A. Mukubwa and J. W. Makokha, "Energy of plasmon-mediated boson-fermion pair condensate in high temperature 
superconductors," Physica B: Condensed Matter, vol. 618, Article ID 413182, 2021.

[36] W. Buckel and R. Kleiner, Superconductivity: Fundamentals and Applications, Wiley-VCH, Weinheim, 2nd edition, 2004.

[37] S. J. Moon, Y. S. Lee, A. A. Schafgans et al., "Infrared Pseudogap in Cuprate and pnictide high-temperature superconductors," Physical Review B, vol. 90, Article ID 014503, 2014.

[38] H. Singh and B. Indu, "Anharmonicity and impurity effects on infrared absorption in high temperature superconductors," International Journal of Physics, vol. 4, no. 2, pp. 43-49, 2016.

[39] C. Lian, Z. A. Ali, and B. M. Wong, "Charge density wave hampers exciton condensation in $1 \mathrm{~T}-\mathrm{TiSe}_{2}$," Physical Review $B$, vol. 100, no. 20, Article ID 205423, 2019.

[40] R. Bianco, L. Monacelli, M. Calandra, F. Mauri, and I. Errea, "Weak dimensionality dependence and dominant role of ionic fluctuations in the charge-density-wave transition of $\mathrm{NbSe}_{2}$," Physical Review Letters, vol. 125, no. 10, Article ID 106101, 2020. 